Accepted by The Astrophysical Journal

\title{
LBQS 0015+0239: A Binary Quasar with Small Angular Separation $^{1}$
}

\author{
Chris D. Impey and Cathy E. Petry \\ Steward Observatory, University of Arizona, Tucson, AZ 85721 \\ cimpey@as.arizona.edu, cpetry@as.arizona.edu \\ Craig B. Foltz \\ Multiple Mirror Telescope Observatory, University of Arizona, Tucson, AZ 85721 \\ cfoltz@as.arizona.edu \\ Paul C. Hewett \\ Institute of Astronomy, Madingley Road, Cambridge CB3 OHA, UK \\ phewett@ast.cam.ac.uk \\ and \\ Frederic H. Chaffee \\ W. M. Keck Observatory, 65-1120 Mamalahoa Highway, Kamuela, HI 96743 \\ fchaffee@keck.hawaii.edu
}

\begin{abstract}
We present spectroscopic observations with Keck/LRIS of LBQS 0015+0239, a pair of quasars at $z=2.45$ with a separation of $\Delta \theta=2.2 \operatorname{arcsec}$ (projected linear distance of $17.8 h_{70}^{-1} \mathrm{kpc}$, for $\Omega_{m}=0.3, \Lambda=0.7$ ). Lensing is an unlikely
\end{abstract}

\footnotetext{
${ }^{1}$ The observations reported here were obtained at the W. M. Keck Observatory, which is operated as a scientific partnership among the California Institute of Technology, the University of California, and the National Aeronautics and Space Administration. The observatory was made possible by the generous financial support of the W. M. Keck Foundation.
} 
interpretation for the images, since the spectra show significant differences in the $\mathrm{N} \mathrm{V}$ and C IV emission line profiles, and there is no luminous galaxy at the anticipated lens position. Rather, we interpret this pair as the highest redshift known example of a binary quasar. The redshift difference of $661 \pm 173 \mathrm{kms}^{-1}$ between the two components is inconsistent with the lensing expectation, but is consistent with the line of sight velocity difference of a bound pair of galaxies. The spectra show associated, narrow metal absorption in highly ionized species of $\mathrm{C}$ and $\mathrm{N}$, with a systemic velocity difference of $492 \pm 6 \mathrm{kms}^{-1}$. It is likely that this absorption arises in a group or cluster of galaxies surrounding the quasar pair. After a thorough search, the Large Bright Quasar Survey (LBQS) is known to contain one gravitational lens and four probable binary pairs. The existence of close binaries is evidence that quasar activity can be triggered by galaxy interactions. We speculate that the close pair LBQS 0015+0239 is a rare example of the pre-merger state of two supermassive black holes.

Subject headings: quasars: individual (LBQS 0015+0239) — quasars: emission lines — quasars: absorption lines — galaxies: interactions

\section{Introduction}

The rare situations of closely-separated quasars at the same redshift are of particular interest for observational cosmology. The paired images occur at a rate of $\sim 1$ in 500 quasars, and they are either the result of gravitational lensing or of physically distinct quasars in a binary system. Many of the pairs (and all of the triples and higher multiplicities) are the result of gravitational lensing by foreground mass distributions. Over 60 lens systems are known (see the review of Surdej \& Claeskens 2002, and the constantly updated list at the CASTLES web site http://cfa-www.harvard.edu/castles/). To be confirmed as a lens, the primary requirements are identical quasar redshifts, and a luminous galaxy at a plausible position and redshift to cause the observed image splitting and brightness ratio (Blandford \& Narayan 1992; Schneider, Ehlers, \& Falco 1992; Kochanek 1995). Almost all of these systems have separations smaller than $\Delta \theta=3$ arcsec. At larger separations, there is a set of about 20 quasar pairs where the interpretation is not secure. A system is best confirmed as a binary if the spectral energy distributions are substantially different, including, for example, cases where one quasar in the pair is radio-loud and the other is radio-quiet. The binary population is important because it leads to the inference that quasar activity can be fuelled by galactic interactions and mergers (Djorgovski 1991; Peng et al. 1999; Kochanek, Falco, \& Muñoz 1999; Mortlock, Webster, \& Francis 1999). 
It is difficult to decide with any certainty whether an individual pair is a lens or a binary. The presence of a luminous, and usually early type, galaxy along the line joining the two images is a good indication of lensing, since the chance probability of such alignment is very small. However, in some cases the available imaging is not deep enough to detect the putative lensing galaxy at all plausible redshifts, and the possibility of "dark" galaxies cannot be entirely ruled out (Hawkins 1997). Image separations of $\Delta \theta=1$ to 3 arcsec are indicative of lensing but not prescriptive, since binaries in a final pre-merger state might have small projected separations. Conversely, the larger separations of $\Delta \theta=3$ to $10 \operatorname{arcsec}$ need not be binaries, since galaxy lensing can get an assist from a surrounding cluster potential (Walsh, Carswell, \& Weymann 1979; Lawrence et al. 1984; Wisotzki et al. 1993). Measurement of out-of-phase but identical fractional flux variations between the two images would be sufficient evidence to declare a lens; but this type of data has not been acquired for any system where the lensing interpretation was not already secure. Another prediction of lensing is achromaticity. However, a clean test for spectral differences is complicated by the generally high degree of similarity of quasar spectra (Francis et al. 1992), the intrinsic variations in quasar continua and broad lines (Small, Sargent, \& Steidel 1997), and the possibility of differential extinction and reddening from a dusty lens (Falco et al. 1999). Spectral differences can also be caused by microlensing, where a stellar mass object can temporarily magnify the continuum of one image relative to the emission lines (Wisotzki et al. 1993; Lewis et al. 1998).

The status of an individual pair can only be evaluated from the ensemble of its observed properties. Several recent papers have attempted to do this. Kochanek, Falco, \& Muñoz (1999) and Peng et al. (1999) both worked primarily with data gathered as part of the CfA/Arizona Space Telescope Lens Survey (CASTLES), and Mortlock, Webster, \& Francis (1999) applied their own criteria to cataloged quasar pairs. These lists are in broad agreement, containing 13, 14, and 16 binaries respectively, after subtracting off the pairs where lenses have subsequently been detected. Two out of the 16 binaries listed by Mortlock et al. are noted as being possibly lensed. With Q 1208+1011, the luminosity and small separation are suggestive of lensing. Recent Hubble Space Telescope observations have failed to detect a lens, but the small separation and high contrast does not allow a sensitive limit (Léhar et al. 2000). Since there is no actual evidence that it is a binary, it is preferable to consider it as a probable lens. In the case of the wider separation pair Q 2345+007, lensing was intially indicated by the strikingly similar spectra (Steidel \& Sargent 1991). However, recent Chandra observations find differences in the overall energy distributions that strongly indicate that this system is a binary (Green et al. 2002). The number of possible binaries increases from 15 to 19 if the objects discovered subsequent to the Mortlock et al. paper are included: CLASS B0827+525 (Koopmans et al. 2000), CTQ 839 (Morgan et al. 2000), the serendip- 
itously discovered LBQS 0103-2753 (Junkkarinen et al. 2001), and LBQS 0015+0239 (this paper).

Another approach is to compare the statistical properties of the population of possible binaries to the properties of confirmed lens systems. The main caveat to this approach is the inhomogeneous nature of the sample of known pairs, which come from a wide variety of radio and optical surveys. The detection of one or both quasars as radio sources provides additional discriminatory power, since the radio-to-optical flux ratios of quasars vary over a wide range, and most quasars are radio-quiet (Hooper et al. 1996; Bischof \& Becker 1997). Kochanek et al. (1999) have used the absence of pairs where both quasars are radio-loud, and the incidence of pairs where one quasar is radio-loud, to argue that essentially all the pairs where no lens is seen are in fact binaries. In addition, the distribution of redshifts, flux ratios and separations of the pairs is different from comparable distributions for confirmed lenses (Mortlock et al. 1999). The spectral similarity of the quasar pairs is suggestive, but not compelling, evidence in the other direction (Peng et al. 1999; Mortlock et al. 1999).

In this paper, we present observations of a small separation quasar pair that we interpret as a probable binary system. The new system is noteworthy because of the high redshift, the small angular separation, and the fact that it comes from a well-studied quasar catalog. Although the Large Bright Quasar Survey (LBQS) has been eclipsed in size by the $2 \mathrm{dF}$ and SDSS quasar surveys (Richards et al. 2001; Croom et al. 2001), it has been the subject of a large amount of follow-up activity, and its completeness has been calibrated with respect to the FIRST survey (Hewett, Foltz \& Chaffee 2001). Most importantly, it has been thoroughly searched for small separation pairs (Hewett et al. 1998). In section 2, we present Keck/LRIS observations which resolve the components of this 2.2 arcsec separation pair. The spectra are noteworthy for the associated heavy element absorption that is seen in each component. In section 3, we present the evidence that LBQS 0015+0239 is a binary rather than a lens. Section 4 contains comments on the incidence of lenses and binaries in the LBQS, and section 5 offers brief comments on the overall population of binary quasars. Conclusions follow in section 6 .

\section{Observations}

All the LBQS quasars have been inspected visually on UK Schmidt Telescope plate material to provide a census of companion objects within a radius of $10^{\prime \prime} 0$ (Hewett et al. 1998). Companions with magnitudes brighter than $B_{J}=21.5-22$, depending on the quality

and depth of the $B_{J}$ plates, have been identified. However, in practice, the physical extent of the high density images of the relatively bright quasars on the plates precludes the detection 
of faint companions closer than $\sim 33^{\prime \prime} 0$ and LBQS 0015+0239 appeared as a single object on the original plate material. A supplementary program of broad band imaging at optical wavelengths, using a variety of telescopes, has produced CCD images of 230 LBQS quasars. The majority of the images were obtained under conditions of good seeing $(\leq 1$.". 0$)$, but are not particularly deep. The companion to LBQS $0015+0239$ was identified in $V R I$ images obtained in 1996 at the $1 \mathrm{~m}$ Jacobus Kapteyn Telescope and the image pair was flagged as a potential gravitational lens/binary quasar.

The quasar pair LBQS $0015+0239$ was observed on the night of 2001 July 21 with the 10m Keck I telescope and the Low Resolution Imaging Spectrograph (Rodgers \& McCarthy 1994). Observations were made in "Red Only" mode. The Red CCD is a 2048x2048 SITe/Tektronix with $24 \mu \mathrm{m}$ pixels, giving $0.215 \mathrm{arcsec} / \mathrm{pix}$ averaged over the field of view. The $600 \mathrm{l} / \mathrm{mm}$ grating was centered just off its $5000 \AA$ blaze at $5310 \AA$ providing wavelength coverage of 4000-6621 $\AA$ with $1.28 \mathrm{~A} /$ pix dispersion and a resolution of $4.2 \AA$. The slit width was 1 arcsec and the slit was aligned with the two images. Three 15 minute exposures were obtained in approximately 0.7 arcsec seeing conditions, which was sufficient to resolve the two images. Flatfields were taken, a flux standard was observed (BD+28 4211$)$, and $\mathrm{HgNeAr}$ and CdZn arc lamps were obtained for both the science and calibration frames.

The data was reduced using standard IRAF ${ }^{2}$ routines. The dual amp readout of the LRIS CCD necessitates the use of the Keck Observatory package wmkolris for overscan and bias subtraction. The flatfielding required an extra step in the determination of the CCD response function because of the discontinuity introduced by the dual amp readout of the chip. Because the standard routines do not allow for discontinuities, we simply fit each half of the chip independently using the standard routines, then joined the two halves to obtain the complete response function. Before extracting the one-dimensional spectra, the three frames are shifted and median combined to eliminate cosmic rays. A variance weighted extraction is performed for each quasar component, including flux from \pm 4 pixels to either side of the peak of the spatial profile. To obtain a summed spatial profile, the final combined image is corrected for geometrical distortion and pixels corresponding to $\lambda \lambda$ 4600-6400 are summed along the dispersion axis using the IRAF task blkavg. All of the major absorption features seen in the combined spectrum are present in the individual 15 minute exposures.

Spectra of the two components of LBQS 0015+0239 are shown in the panels of Figure 1. Prominent emission lines are labelled, and narrow absorption lines are marked along the continuum. The selection of absorption lines is based on software that uses a similar detection

\footnotetext{
${ }^{2}$ The Image Reduction and Analysis Facilities package is distributed by NOAO, which is operated by AURA Inc. under contract to the National Science Foundation.
} 
strategy to that used by the Quasar Absorption Line Key Project (Schneider et al. 1993). The basic features of the software are given in Petry, Impey, \& Foltz (1998), and additional details can be found in Petry et al. (2002). The continuum is modelled with a spline fit that adequately follows the rapidly changing flux in the vicinity of the broad emission lines. Absorption features are selected using an interative technique that assumes unresolved lines and can deblend multiple Gaussian profiles in regions of complex absorption.

Tables 1 and 2 list the significant absorption lines detected in the two components A and B of LBQS 0015+0239. Significance is defined in two ways: $S L_{f i t}$ which reflects how well the absorber is fit by the software, and $S L_{\text {det }}$ which refers to the detection limit of the data. $S L_{f i t}$ is simply the ratio of the fitted equivalent width to its error, $W / \sigma_{f i t} . S L_{\text {det }}$ is different in that its denominator, $\sigma_{\text {det }}$, is the $1 \sigma$ limiting equivalent width, which is a function of wavelength and is computed using the flux error. To obtain a reliable list of absorbers, we use a joint criterion for declaring significant lines: $S L_{f i t} \geq 3$ and $S L_{\text {det }} \geq 4$. Additionally, some lines in blended regions can have very low $S L_{f i t}$ even though the detection limit of the data may be low, resulting in a higher $S L_{\text {det }}$. Therefore, we include any line with $S L_{\text {det }} \geq 6$. The RMS error in the wavelength calibration is $0.07 \AA$, although the absolute error is higher shortward of $4500 \AA$, where there are very few arc lines. Differential wavelength errors are smaller, but in practice the precision of measuring wavelength differences between the spectra is limited by the error on the fitted central wavelength of each feature, as can be seen in Table 1.

The difference in signal-to-noise ratio between the two spectra reflects the fact that component A is nearly ten times brighter than component B. Each spectrum shows strong Ly $\alpha$, S IV, and C IV emission, with the red end of the Ly $\alpha$ forest seen below $4200 \AA$. N V is strong in the wings of Ly $\alpha$ in component A and virtually absent in component B. Strong and narrow associated absorption lines are seen eating into the Ly $\alpha, \mathrm{N}$ V, Si IV, and C IV emission features. The redshifts of the quasars were measured by cross-correlation with the SDSS composite, using the IRAF routine fxcor. The results are $z=2.4747 \pm 0.0043$ for component A and $z=2.4831 \pm 0.0044$ for component B. The difference of $\Delta v=720 \pm 380 \mathrm{kms}^{-1}$ differs from zero at the $2 \sigma$ level. Redshifts from cross-correlation are reliable because they use the full information in the spectra. However, the two components of LBQS 0015+0239 are more similar to each other than they are to the SDSS composite (as indicated by a higher correlation amplitude), so the most precise measure of the velocity difference comes from the cross-correlation between A and B. The cross-correlation result is $\Delta v=661 \pm 173 \mathrm{kms}^{-1}$, which is formally different from zero at the $4 \sigma$ level. The amplitude of the cross-correlation is 0.92 , indicating the high degree of spectral similarity of the two components.

The major emission lines in each of the two components are stronger, by factors of 1.4 
$(\mathrm{Ly} \alpha / \mathrm{N} \mathrm{V}$ ), 2.3 (Si IV/O IV), 2.0 (C IV), and 3.5 (He II) than the rest equivalent widths for the SDSS composite spectrum (Vanden Berk et al. 2001). The agreement between the two components is in all cases better than 35\%. Peng et al. (1999) found that the spectral similarities of the binary pairs were significantly greater than would be expected for an equivalent sample of randomly selected quasars (but see also Mortlock et al., who reach a different conclusion for two of the LBQS pairs). The spectra of the two components of LBQS 0015+0239 raise again the question of how two quasars with an order of magnitude different luminosity could have such similar properties on parsec scales.

The associated absorbers have a systematic velocity difference of $492 \pm 6 \mathrm{kms}^{-1}$ between the two components. This number is based on a velocity error weighted combination of the offsets between $\mathrm{A}$ and $\mathrm{B}$ of four different species, listed in Table 3. The two components of C IV are combined into a single error-weighted offset before averaging with the other three species. Table 1 also lists Ly $\alpha$ absorbers seen at the short wavelength ends of the spectra, with 16 lines detected in component A, and 9 lines detected in component B. There are 5 coincident absorbers, where the wavelengths match within $2.5 \sigma$ of the measurement errors. An additional 3 absorbers in B are resolved into 2 components each in A, where the average wavelength in A matches within the measurement errors to B. The non-detection of an absorber in A to pair to the remaining unmatched line in B is most probably due to the uncertain continuum fit on the wing of Ly $\alpha$ emission in A. The strengths of these coincident Ly $\alpha$ absorbers are consistent with being equal, given the different signal-to-noise ratios and equivalent width limits of the two spectra except for the two matches on the wing of Ly $\alpha$ emission. This result is expected, since low column density hydrogen at this redshift has a coherence length much larger than the transverse separation of these sightlines (Dinshaw et al. 1994; Fang et al. 1996).

\section{Interpretation of LBQS $0015+0239$}

As discussed in the introduction, it can be difficult to directly confirm an individual quasar pair as either a lens system or a binary. In this case, the systemic velocity difference of $661 \pm 173 \mathrm{kms}^{-1}$ between the two components differs from the lensing expectation at the $3.8 \sigma$ level. Additional evidence for a binary can come when one image of a quasar pair is a stronger radio source than the other (Kochanek et al. 1999). However, LBQS 0015+0239 is not in the area of sky covered by FIRST, and NVSS only provides an upper limit to the combined flux density for both quasars of $2.5 \mathrm{mJy}$ at $1.4 \mathrm{GHz}$ (Condon et al. 1998). The second way to search for achromaticity uses the optical spectra. The upper panel of Figure 2 presents the spectra of the two components with an arbitrary scaling and offset to illustrate 
the spectral similarity. The lower panel of Figure 2 shows the ratio of component A to component $\mathrm{B}$ where an interpolated continuum was used to replace the narrow associated absorption lines in each spectrum. The flux ratio is roughly a factor of ten with no evidence for any strong change with wavelength. We fit a straight line to the flux ratio, after truncating the data below $4400 \AA$, since the Ly $\alpha$ forest is heavily absorbed and the flux ratio there will depend on resolution and the signal-to-noise ratio. The slope is zero to within the error of the fit. There are two systematic and significant departures from a flux ratio of ten. The first occurs just redward of $\operatorname{Ly} \alpha$, reflecting the much stronger $\mathrm{N}$ V emission line of component A. The other is centered on C IV emission, reflecting different line profiles for the two components. The $\mathrm{N}$ V difference between $\mathrm{A}$ and $\mathrm{B}$ supports the interpretation of the pair as a binary, since this difference is much larger than the temporal variations seen in individual quasars on timescales appropriate to a time delay under the lens hypothesis (Small et al. 1997).

Another approach is to look for a lensing galaxy. No deep image has been taken of LBQS 0015+0239 under good enough seeing conditions to put a constraint on a lens. However, the Keck spectra are well-resolved spatially, so they offer the opportunity of a measurement. The spectra were linearized on the final coadded image and then collapsed in wavelength to produce a 1D spatial crosscut along a line joining components A and B. Figure 3 shows the resulting spatial profile. A Gaussian fit to the two component centers in the crosscut yields an image separation of 10.2 pixels or $2.20 \pm 0.03$ arcsec. We choose a wavelength range of 4600-6400 $\AA$ for the crosscut, because it approximates the $V$ passband and it avoids the edges of the CCD where the spectra flare out slightly due to defocussing. The bright component $\mathrm{A}$ has a magnitude of $m_{B}=18.7$ on the discovery plate material (Hewett, Foltz, \& Chaffee 1995). Direct photometry yields $V=19.4$ for component A and $V=21.9$ for B (Hewett \& Foltz 2002, in preparation). Under the lensing hypothesis, the most probable lens redshift is $z=0.44$ at a position between A and B but $90 \%$ of the way towards the faint component (C. Y. Peng 2002, private communication). We assume an early type galaxy with a singular isothermal sphere halo, since known lenses generally fit this description (Keeton, Kochanek, \& Falco 1998). At $z=0.44$, the velocity dispersion would be $247 \mathrm{kms}^{-1}$. Scaling from a local Faber-Jackson relationship predicts a lens luminosity of $1.6 L_{*}$, which is $V=20.7$ after (passively) de-evolving the light to $z=0.44$.

A simple lens model therefore sets the expectation that the lens should be located between components A and B, and it should be much brighter than the faint component. Even without deep imaging, our spectroscopy is sufficient to rule this out. A Gaussian is actually a poor fit to the wings of the image profile in Figure 3. Instead, we omit the peak of the profile and fit a Gaussian to the lower slope and wing of component $\mathrm{A}$ in the direction away from component B, since a lens will not contribute significant light at that position. 
Assuming symmetry, we fold this profile about the peak of $\mathrm{A}$ and use that shape for the wing of $\mathrm{A}$ in the direction of $\mathrm{B}$. Component $\mathrm{B}$ is modelled by the same shape scaled by the ratio of peak fluxes. This procedure accounts for essentially all of the flux between the peaks of the two components, resulting in a strong limit on residual lens light. We can use the results to calculate the cross-contamination of the spectra presented in Figure 1: $6 \%$ of $\mathrm{B}$ is light from $\mathrm{A}$ and $0.03 \%$ of $\mathrm{A}$ is light from $\mathrm{B}$. The upper limit on the amount of residual extended light at any position between $\mathrm{A}$ and $\mathrm{B}$ corresponds to $V>22.8$.

However, in a realistic lensing scenario, the lens would be 10 times closer to B than to A. The amount of residual extended light on or near the fainter component $\mathrm{B}$ gives a much more stringent upper bound of $V>24.4$. This constraint is only weakened in the unlikely scenario that the lens is compact enough to be unresolved and is positioned exactly in front of component B. However, the flux ratio in Figure 1 shows no achromatic signature from the admixture of red light from an early type galaxy, and in fact, the $4000 \AA$ break would be seen at any lens redshift in the range $0.08<z<0.62$. Therefore, any putative lens galaxy might be at least 30 times underluminous for its mass compared to galaxies in confirmed lens systems. Relative to the stellar mass-to-light ratio of an early type galaxy, this corresponds to a limits of $(M / L)_{V} \gtrsim 200$. However, since the probability distribution for the "most probable" lens redshift is fairly broad, these limits could be a factor of 3-5 weaker. Since there is no other compelling evidence for massive dark galaxies, we prefer to interpret LBQS 0015+0239 as a binary quasar.

\section{Incidence of Binaries and Lenses in the LBQS}

The LBQS contains 1067 quasars, selected according to their spectral energy distributions on objective prism plates from the UK Schmidt Telescope (Hewett, Foltz \& Chaffee 1995). The survey covers an apparent magnitude range of $16.0 \leq m_{B} \leq 18.85$, a redshift range of $0.2 \leq z \leq 3.4$, and is known to be no more than about $10 \%$ incomplete fainter than $m_{B}=16.5$ based on a comparison with the FIRST radio survey (Hewett, Foltz, \& Chaffee 2001). The LBQS has been subject to a specific search for companions within 10 arcsec of each quasar, down to level of $m_{B}=21.5$ (Hewett et al. 1998). The ongoing $2 \mathrm{dF}$ and SDSS quasar surveys will eventually produce substantial new samples of close pairs. However, in each case, extensive follow-up observations are required. Both surveys use fibers which preclude the spectroscopic observation of pairs with separations under 20 arcsec. In addition, pairs less than 6 arcsec (for the $2 \mathrm{dF}$ ) and 1.5 arcsec (for SDSS) have images that are merged on the original survey material. At present, the LBQS is the largest sample that has been closely inspected for pairs. 
There are five quasar pairs with separations under 10 arcsec in the LBQS. One pair is a confirmed gravitational lens system, and the other four are probable binaries. Hewett et al. (1998) described the follow-up spectroscopy of plausible candidates for quasar companions. Quasar pairs with widely discrepant redshifts are expected to occur by chance. Given the surface density of quasars down to $m_{B}=21.5$, Hewett et al. (1998) predicted 1.6 quasar pairs with unrelated redshifts in the LBQS, which is consistent with the one known (Hewett et al. 1994). LBQS 1009-0252 is confirmed as a lens system at $z=2.74$, primarily due to the detection of a lens galaxy at an appropriate location between the two components (Claeskens et al. 2001). In addition, there is metal line absorption in the two spectra at $z=0.87$, which is close to the lens redshift as predicted from a model and as estimated from the photometric properties. There is also a third image which is a quasar at $z=1.62$, so this system qualifies as a second quasar pair with discrepant redshifts. The largest surveys for gravitational lenses are radio surveys carried out at Jodrell Bank and the VLA (Patnaik et al. 1992; Myers et al. 1995; Phillips et al. 2001). With 18 lenses from 11,670 radio sources, roughly half of which are doubles, the presence of only one lens in the LBQS is consistent with expectations.

LBQS $1429-0053$ is a pair at $z=2.08$ with an angular separation of $\Delta \theta=5.1$ arcsec (Hewett et al. 1989). Under the lensing hypothesis, this separation is most likely to be caused by a single massive galaxy with an assist from a surrounding cluster. However, Hewett et al. find no luminous object at the expected lens position, with a lower limit of $2000<(M / L)<4000$, depending on cosmology. In addition, the spectra show a difference of $\sim 50 \%$ in the relative strength of the Ly $\alpha-\mathrm{N}$ V complex, therefore LBQS 1429-008 is a probably binary. LBQS $2153-2056$ is a pair at $z=1.85$ with an angular separation of $\Delta \theta=7.8 \operatorname{arcsec}$ (Hewett et al. 1998). Available imaging is not deep enough to place a sensitive limit on a deflector, but the fact that only one member of the pair is a BAL quasar argues against lensing unless the BAL clouds are extremely small (see Mortlock et al. 1999). LBQS 0103-2753 is by far the smallest separation binary known (Junkkarinen et al. 2001), at $z=0.85$ with a separation of $\Delta \theta=0.3$ arcsec. The binary interpretation is secure because the spectra are very different (and one quasar has BAL features). Under the lensing hypothesis, the time delay is $\sim 10$ days, and spectral variations sufficient to explain the differences between two components' spectra are never observed in quasars on such a short timescale. As we have argued in this paper, LBQS 0015+0239 is a strong candidate for a fourth binary quasar in the LBQS.

The statistical properties of the LBQS pairs provide additional evidence that they are in fact binaries. Three out of four have flux ratios of ten or greater, versus only 3 out of 24 two-image lenses with data tabulated by CASTLES. Lack of spectral similarity provides an additional argument. The primary of the serendipitously discovered close pair 
LBQS 0103-2753 was selected as a BAL quasar, but the companion has a very different, and unexceptional, spectrum with no strong absorption. Mortlock et al. (1999) used principal compnents analysis to show that LBQS 1429-0053 and LBQS 2153-2056 are as dissimilar as any quasar pairs randomly selectly from the LBQS. This would not be expected if either pair were lensed.

The pair that is the subject of this paper is unusual in that both quasars show associated absorption. This is noteworthy, given that fewer than 10\% of LBQS quasars have this attribute. Associated absorbers are usually defined as metal systems within $\Delta v= \pm 5000$ $\mathrm{kms}^{-1}$ of the emission redshift. Such absorption might be intrinsic and related to the broad absorption line (BAL) phenomenon, or extrinsic and caused by galaxies in a cluster containing the quasar (Foltz et al. 1988). Evidence for the latter interpretation comes from abundance studies. Nine out of ten associated systems have $(N / C)$ ratios below solar abundance, while BAL regions have $(N / C)$ ratios of 9-10 times solar abundance (Turnshek et al. 1996; Franceschini \& Gratton 1997). This new pair also supports the extrinsic explanation of associated absorption, as the redshift difference of $492 \pm 6 \mathrm{kms}^{-1}$ is consistent with a group or cluster environment, and the fact that the pair is a binary system shows that there are at least two massive galaxies in this region of space.

To date, four binary quasars and a single gravitational lens have been discovered in the LBQS. The incidence of binary pairs is difficult to predict a priori, but it is worth asking what is expected for the rate of lensing in a survey like the LBQS. Maoz et al. (1993) conducted the largest optical lens survey that was sensitive to the angular separations of essentially all known lens systems. The survey found 5 lenses out of 502 quasars surveyed (Maoz gave a range of 3-6, so this count includes $0957+561$ but excludes the unconfirmed candidate UM 1120+0154), for a lensing rate of 1\%. For the purposes of this paper, we will not carry out a full analysis of the predicted lensing rate of the LBQS, but we can easily give an estimate relative to the Maoz HST snapshot survey.

The predicted number of multiply imaged quasars for any sample is found by calculating the probability of each quasar being lensed and summing over these probabilities for the entire sample. The probability that an individual quasar is lensed is the product of its optical depth to lensing and its magnification bias (Turner, Ostriker, \& Gott 1984; Fukugita \& Turner 1991). Pei (1995) has published maps of magnification bias in redshift and apparent blue magnitude, assuming a double power law model for the quasar luminosity function and a lens population that is composed of compact objects, individual galaxies, and clusters. The shape of the magnification bias contours is insensitive to quite varied models of the lensing population. Taking the $50 \%$ and $90 \%$ bounds in redshift and magnitude for the two samples, it turns out that the mean magnification bias is low for both the LBQS and the HST 
snapshot survey, just under $1 \%$. The most substantial difference between the two samples is the mean redshift, about 1.2 for the LBQS and 2.0 for the HST snapshot survey. For a $\Lambda$-dominated cosmology, the lensing optical depth scales as $\tau \sim z^{3}$ (Bahcall et al. 1992). Thus, the lensing rate should be 4-5 times lower for the LBQS than for the HST snapshot survey, consistent with the single lens that has so far been identified.

Despite this argument, it is admitted that the predictions of lensing rates are uncertain, and the purely observational limits on additional lenses in the LBQS are poor. The companion search of Hewett et al. (1998) was only sensitive to separations greater than 3 arcsec (although equal brightness doubles as close as 2 arcsec could have been identified). The LBQS search was therefore insensitive to about $90 \%$ of the lens image separations from a radio survey such as CLASS. The presence of a few more small separation lenses in the LBQS cannot be ruled out.

\section{The Population of Binary Quasars}

The LBQS contains four binary pairs with projected separations less than $100 h_{70}^{-1} \mathrm{kpc}$ (plus the previously mentioned lens which is, of course, a single quasar). Since the newly discovered LBQS 0015+0239 has smaller angular separation than the 3-10 arcsec completeness range of the search by Hewett et al. (1998), and since the 0.3 arcsec pair LBQS 0103-2753 was discovered serendipitously (Junkkarinen et al. 2001), it is worth asking how many more small separation binaries might be lurking in the LBQS. Maoz et al. (1993) performed a snapshot survey of 498 quasars with $z>1$ and found no binaries with separations below 3 arcsec. They could have detected binaries as close as 0.3 arcsec, and with flux ratios of at least 4:1. Thus, the single close LBQS binary is consistent with the null result of Maoz et al., and Poisson statistics suggest that there are unlikely to be more than two additional very close binaries in the LBQS.

Over the order of magnitude larger range of scales from 10 to 100 arcsec, there are no LBQS pairs with similar redshifts. Unfortunately, this null result is not a strong constraint, since the deep companion search of Hewett et al. (1998) applied over this angular range would have been been sensitive to 30 times as many quasars. As noted originally by Djorgovski (1991), the high incidence of close pairs is strong circumstantial evidence that quasar activity can be triggered by direct interactions. Extrapolating the observed quasar correlation function down to these small physical scales leads to a prediction of $\sim 0.01$ quasar

pairs within $100 h_{70}^{-1} \mathrm{kpc}$, compared to the four observed. A similar scaling of the correlation function predicts 20 times as many pairs between 10 and $100 \operatorname{arcsec}$ as between 3 and 10 arcsec, or $\sim 40$ wide pairs. 
We adopt the pair fraction of the LBQS (4/1055) as the probability that a quasar is a member of a binary, $P_{\text {binary }}=4 \times 10^{-3}$. The LBQS probability only applies out to 10 arcsec, or a transverse separation of $\sim 100 h_{70}^{-1} \mathrm{kpc}$. If the interaction-induced quasar activity occurs in the advanced stages of a merger process, then we anticpate that the probability summed to much larger separation scales will not be much larger (Barnes \& Hernquist 1996). If the radio-loud fraction of optically selected quasars is $f_{\text {rad }}$ and we assume that binarism does not affect radio properties, then the relative numbers of $O^{2}, O^{2} R$, and $O^{2} R^{2}$ binaries (ie. those where both, one, and neither quasar are radio-loud) are in the ratio $\left(1-f_{\text {rad }}\right)^{2}$ : $2 f_{\text {rad }}\left(1-f_{\text {rad }}\right): f_{\text {rad }}^{2}$ (Kochanek et al. 1999). Updating the Mortlock et al. list (see comments in the Introduction) gives $12 O^{2}$ pairs. Taking $f_{\text {rad }} \simeq 0.1$, as is appropriate for FIRST or NVSS detections above 1-3 mJy, the predictions are $0.1 O^{2} R^{2}$ pair, which is consistent with none seen, and $2.2 O^{2} R$, which is consistent with two seen.

Recent radio lens surveys are an order of magnitude larger than the LBQS. Also, the surface density of radio sources is low enough that it is much more feasible to search for pairs out to larger angular separations. So far, only one radio-loud binary $\left(O^{2} R^{2}\right)$ has been found among 15,000 CLASS sources (Koopmans et al. 2000), and none have been found among 9100 FIRST sources (Ofek et al. 2002). The only other known $O^{2} R^{2}$ binary is MG 0023+171 (Hewitt et al. 1987). It is entirely plausible that several more $O^{2} R^{2}$ binaries remain to be found since the radio morphologies of each component may be quite different, thus eluding the selection designed to catch lens candidates. Using the LBQS estimate of $P_{\text {binary }}$, the total number of $O^{2} R$ binaries present in the CLASS and FIRST surveys out to $\Delta \theta=10$ arcsec should be $\sim 57$ and $\sim 35$, respectively. The number where both components are radio-loud is a factor of $f_{\text {rad }}$ smaller, or $\sim 3$ and $\sim 4$, respectively. The numbers should also be increased to account for the larger angular scales of the CLASS (15 arcsec) and FIRST (30 arcsec) surveys, assuming that the association probability scales according to the quasar-galaxy correlation function. The smaller size of the FIRST survey is outweighed by the deeper radio limit and the larger angular search radius. For the FIRST survey, we predict $\sim 15 O^{2} R$ binaries. We predict $\sim 100$ and $\sim 400$ binaries in the $2 \mathrm{dF}$ and SDSS optical quasar surveys, but note that substantial spectroscopic followup will be needed to identify the second (fainter) components in each survey.

LBQS 0015+0239 is the third smallest separation binary quasar known. Kochanek et al. (1999) have shown that number of binaries is consistent with the quasar-galaxy correlation function at small scales. Mortlock et al. (1999) deprojected the observed separations of binary pairs to yield a broad distribution peaking at $\sim 50 h_{70}^{-1} \mathrm{kpc}$, a declining tail out to large separations, and a possible deficit at small separations. Despite the lack of comprehensive follow-up of wide pairs from radio surveys, the decline at large separations appears to be real and is indicative of an interaction trigger for the nuclear activity. LBQS 0015+0239 and 
the other two binary quasars discovered since Mortlock et al. neatly fill in their "hole," so the separation distribution may be flat below $50 h_{70}^{-1} \mathrm{kpc}$. Given this type of distribution, the 0.3 arcsec LBQS pair discovered by Junkkarinen et al. (2001) is far more likely to be a real 2-3 $h_{70}^{-1}$ binary than a chance projection of a $\sim 40 h_{70}^{-1}$ kpc binary. LBQS quasars are typically found in massive galaxies (Hooper, Impey \& Foltz 1997), where the dynamical decay timescale of the binary orbits is close to a Hubble time and much longer than active timescale of quasars accreting at near the Eddington limit (Binney \& Tremaine 1987). Thus, LBQS 0015+0239 may have been triggered by the much faster settling process with the merged halo (Barnes \& Hernquist 1996). Deep imaging of this pair, and many of the other binaries, should reveal heavily distorted host morphologies.

\section{Conclusions}

We have carried out Keck/LRIS spectroscopy of the 2.2 arcsec separation quasar pair LBQS 0015+0239. These data, plus a consideration of the other pairs in the LBQS, leads to the following conclusions:

1. There are three pieces of evidence that support the interpretation that the pair is a binary system. The velocity difference between the components is inconsistent with the lensing expectation but consistent with a bound pair of massive galaxies. There are substantial differences in $\mathrm{N} \mathrm{V}$, and to a lesser extent C IV, emission line strengths between the two components. A spatial cut along the line connecting the pair in the equivalent of the $V$ band leads to the conclusion that any lensing galaxy must be at least thirty times less luminous than an elliptical galaxy that could account for the image separation.

2. Narrow associated absorption due to metal lines is seen in each component, with a velocity difference of $492 \pm 6 \mathrm{kms}^{-1}$. Since the associated absorption phenomenon is only seen in about $10 \%$ of LBQS spectra, its presence in these two quasars supports the idea that the absorption arises in a group or cluster environment that includes the binary pair.

3. There are four probable binary pairs in the LBQS, so the incidence rate is $4 \times 10^{-3}$, several times higher than the lensing rate. The large radio surveys being used to select gravitational lenses probably contain 15-20 unrecognized binary pairs, where only one of the members is radio-loud. LBQS $0015+0239$ has a projected transverse separation of only 17.8

$h_{70}^{-1} \mathrm{kpc}$ and is the highest redshift binary quasar known. It may represent the short-lived phase prior to the coalescence of two supermassive black holes, in which case deep imaging of the pair should reveal a disturbed morphology for the host galaxies. 
We are grateful to the excellent staff of the W. M. Keck Observatory for facilitating these observations. We benefited from useful discussions on the debate over lensing versus binarism with Chris Kochanek, Ian Browne, and Chien Peng. We thank the referee for a number of useful suggestions. CDI and CBF acknowledge support from the NSF under award AST-9803072. 


\section{REFERENCES}

Bahcall, J. N., Maoz, D., Doxsey, R., Schneider, D. P., Bahcall, N. A., Lahav, O., \& Yanny, B. 1992, ApJ, 387, 56

Barnes, J., \& Hernquist, L. 1996, ApJ, 471, 115

Binney, J., \& Tremaine, S. 1987, Galactic Dynamics, Princeton University Press: Princeton

Bischof, O. B., \& Becker, R. H. 1997, AJ, 113, 2000

Blandford, R. D., \& Narayan, R. 1992, ARA\&A, 30, 311

Claeskens, J.-F., Khmil, S. V., Lee, D. W., Sluse, D., \& Surdej, J. 2001, A\&A, 367, 748

Condon, J. J., Cotton, W. D., Greisen, E. W., Yin, Q. F., Perley, R. A., et al. 1998, AJ, 115,1693

Croom, S. M., Smith, R. J., Boyle, B. J., Shanks, T. \& Loaring, N. S. 2001, MNRAS, 322, L29

Dinshaw, N., Impey, C. D., Foltz, C. B., Weymann, R. J., \& Chaffee, F. C. 1994, ApJ, 437, L87

Djorgovski, S. 1991, in ASP Conference Series Vol. 21 on the Space Distribution of Quasars, ed. D. Crampton, ASP: San Francisco, p. 349

Falco, E. E., Impey, C. D., Kochanek, C. S., Léhar, J., McLeod, B. A. et al. 1999, ApJ, 523, 617

Fang, Y., Duncan, R. C., Crotts, A.P S., \& Bechtold, J. 1996, ApJ, 462, 77

Foltz, C. B., Chaffee, F. H., Weymann, R. J., \& Anderson, S. J. 1988, in QSO Absoprtion Lines: Probing the Universe, eds. C. Blades, D. turnshek, \& C. Norman, Cambridge: Cambridge University Press, p. 66

Franceschini, A., \& Gratton, R. 1997, MNRAS, 286, 235

Francis, P. J., Hewett, P. C., Foltz, C. B., \& Chaffee, F. C. 1992, ApJ, 398, 476

Green, P. J., Kochanek, C. S., Siemiginowska, A., Kim, D.-W., Markevitch, M. et al. 2002, ApJ, in preparation

Hawkins, M. R. S. 1997, A\&A, 328, L25

Hewett, P. C., Webster, R. L., Harding, M. E., Jedrzejewski, R. I., Foltz, C. B., et al. 1989, ApJ, 346, L61

Hewett, P. C., Irwin, M. J., Foltz, C. B., Harding, M. E., Corrigan, R. T., Webster, R. L., \& Dinshaw, N. 1994, AJ, 108, 1534

Hewett, P. C., Foltz, C. B., \& Chaffee, F. H. 1995, AJ, 122, 518 
Hewett, P. C., Foltz, C. B., Harding, M. E., \& Lewis, G. F. 1998, AJ, 115, 383

Hewett, P. C., Foltz, C. B., \& Chaffee, F. H. 2001, ApJ, 122, 518

Hewitt, J. N., Turner, E. L., Lawrence, C. R., Schneider, D. P., Gunn, J. E., et al. 1987, ApJ, 321, 706

Hooper, E. J., Impey, C. D., Foltz, C. B., \& Hewett, P. C. 1996, ApJ, 473, 746

Hooper, E. H., Impey, C. D., \& Foltz, C. B. 1997, ApJ, 480, L95

Fukugita, M., \& Turner, E. L. 1991, MNRAS, 253, 99

Junkkarinen, V., Shields, G. A., Beaver, E. A., Burbidge, E. M., Cohen, R. D. et al. 2001, ApJ, 549, L155

Keeton, C. R., Kochanek, C. S., \& Falco, E. E. 1998, ApJ, 509, 561

Kochanek, C. S. 1995, ApJ, 453, 545

Kochanek, C. S., Falco, E. E., \& Muñoz, J. A. 1999, ApJ, 510, 590

Koopmans, L. V. E., de Bruyn, A. G., Fassnacht, C. D., Marlow, D. R., Rusin, D. et al. 2000, A\&A, 361, 822

Lawrence, C. R., Schneider, D. P., Schmidt, M., Bennett, C. L., Hewitt, J. N. et al. 1984, Science, 223, 46

Léhar, J., Falco, E. E., Kochanek, C. S., McLeod, B. A., Mũnoz, J. A. et al. 2000, ApJ, 536, 584

Lewis, G. F., Irwin, M. J., Hewett, P. C., \& Foltz, C. B., 1998, MNRAS, 295, 573

Maoz, D., Bahcall, J. N., Schneider, D. P., Bahcall, N. A., Djorgovski, S. 1993, ApJ, 409, 28

Morgan, N. D., Burley, G., Costa, E., Maza, J., Persson, S. E. et al. 2000, AJ, 119, 1083

Mortlock, D. J., Webster, R. L., \& Francis, P. J. 1999, MNRAS, 309, 836

Myers, S. T., Fassnacht, C. D., Djorgovski, S. G., Blandford, R. D., Matthews, K. et al. 1995, ApJ, 447, L5

Ofek, E. O., Maoz, D., Prada, F., Kolatt, T., and Rix, H.-W. 2002, MNRAS, in press

Patnaik, A. R., Browne, I. W. A., Wilkinson, P. N., \& Wrobel, J. M. 1992, MNRAS, 254, 655

Pei, Y. C. 1995, ApJ, 440, 485

Peng, C. Y., Impey, C. D., Falco, E. E., Kochanek, C. S., Léhar, J. et al. 1999, ApJ, 524, 572

Petry, C. E., Impey, C. D., \& Foltz, C. B. 1998, ApJ, 494, 60 
Petry, C. E., Impey, C. D., Katz, N., Weinberg, D. H., \& Hernquist, L. E. 2002, ApJ, in press

Phillips, P. M., Browne, I. W. A., Jackson, N. J., Wilkinson, P. N., Mao, S. et al. 2001, MNRAS, 328, 1001

Richards, G. T., Fan, X., Schneider, D. P., Vanden Berk, D. E., Strauss, M. A., et al. 2001, AJ, 121, 2308

Rodgers, J. M., \& McCarthy, J. K. 1994, SPIE, 2198, 1096

Schneider, P., Ehlers, J., \& Falco, E. E. 1992, Gravitational Lenses, Berlin: Springer-Verlag

Schneider, D. P., Hartig, G. F., Jannuzi, J. P., Kirhakos, S., Saxe, D. H., et al. 1993, ApJS, 87,45

Small, T. A., Sargent, W. L. W., \& Steidel, C. C. 1997, AJ, 114, 2254

Steidel, C. C. \& Sargent, W. L. W. 1991, AJ, 102, 1610

Surdej, J., \& Claeskens, J.-F. 2002, ARA\&A, in press

Turner, E. L., Ostriker, J. P., \& Gott, J. R. 1984, ApJ, 284, 1

Turnshek, D. A., Kopko, M., Minier, E., Noll, D, Espey, D. R., \& Weymann, R. J. 1996, ApJ, 463, 110

Vanden Berk, D. E., Richards, G. T., Bauer, A., Strauss, M. A., Schneider, D. P. et al. 2001, AJ, 122,549

Walsh, D., Carswell, R. F., \& Weymann, R. J. 1979, Nature, 279, 381

Wisotzki, L., Kohler, T., Kayser, R., \& Reimers, D. 1993, A\&A, 278, L15 


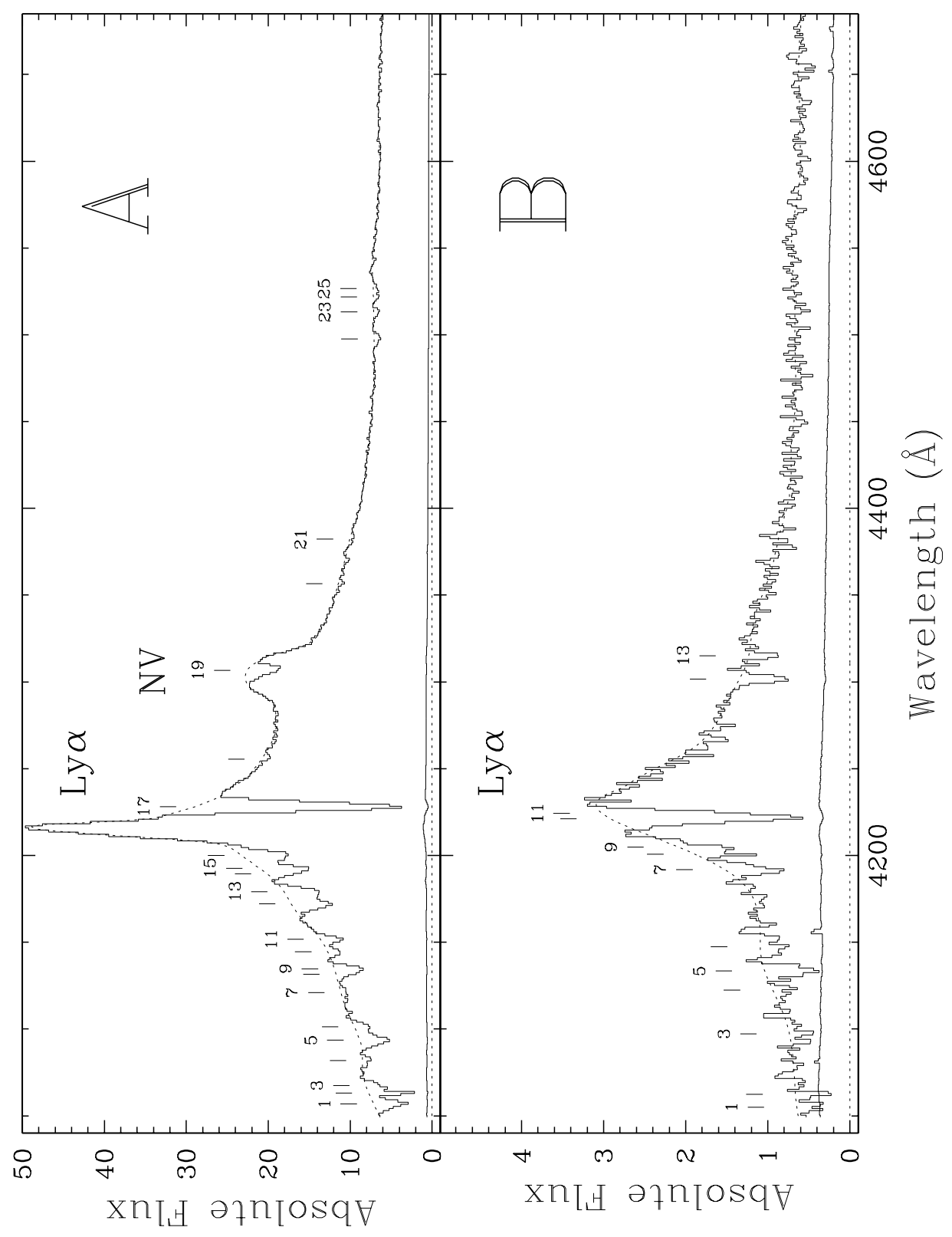

Fig. 1. - Keck/LRIS spectra of the quasar pair LBQS 0015+0239, with $4.2 \AA$ resolution. Cross correlation with SDSS quasar composite gives redshifts of $z=2.4747$ for component A and $z=2.4831$ for component B. The dotted line is the fitted continuum, prominent emission features are labelled, and all significant absorption lines are marked with every other one numbered. The lower curve shows the $3 \sigma$ flux error. The flux is in units of $10^{-17}$ ergs $\mathrm{s}^{-1} \mathrm{~cm}^{-2} \AA^{-1}$. 


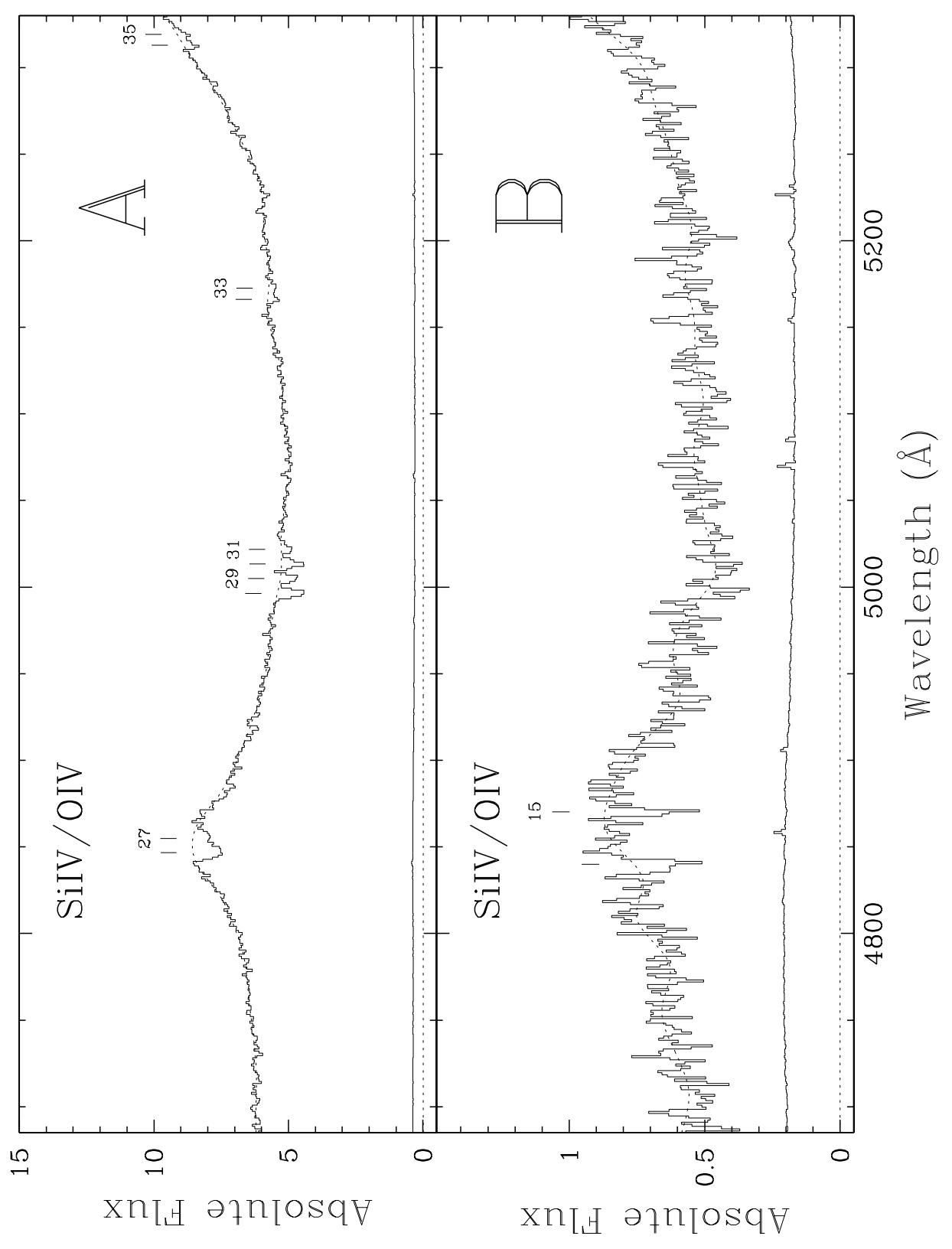




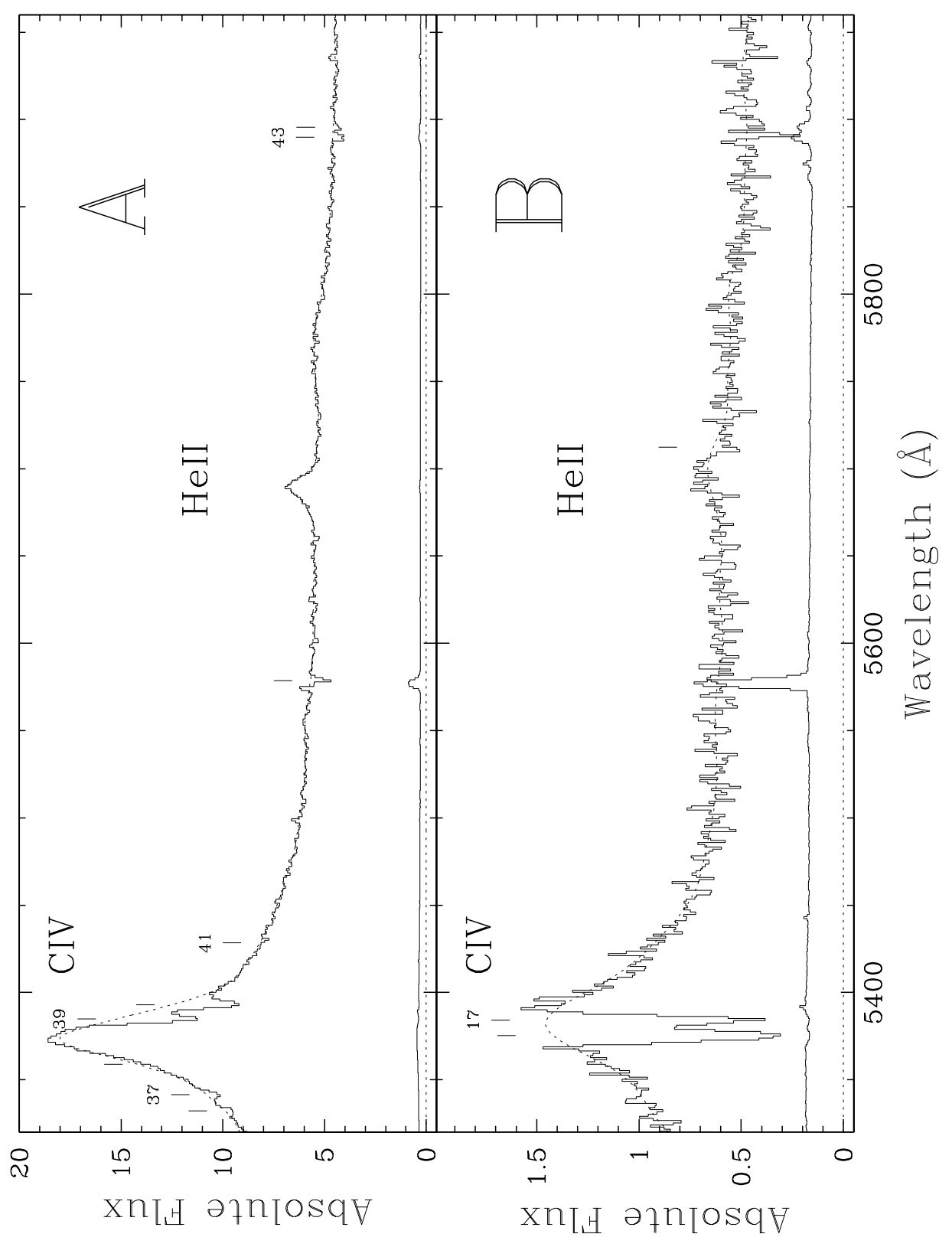




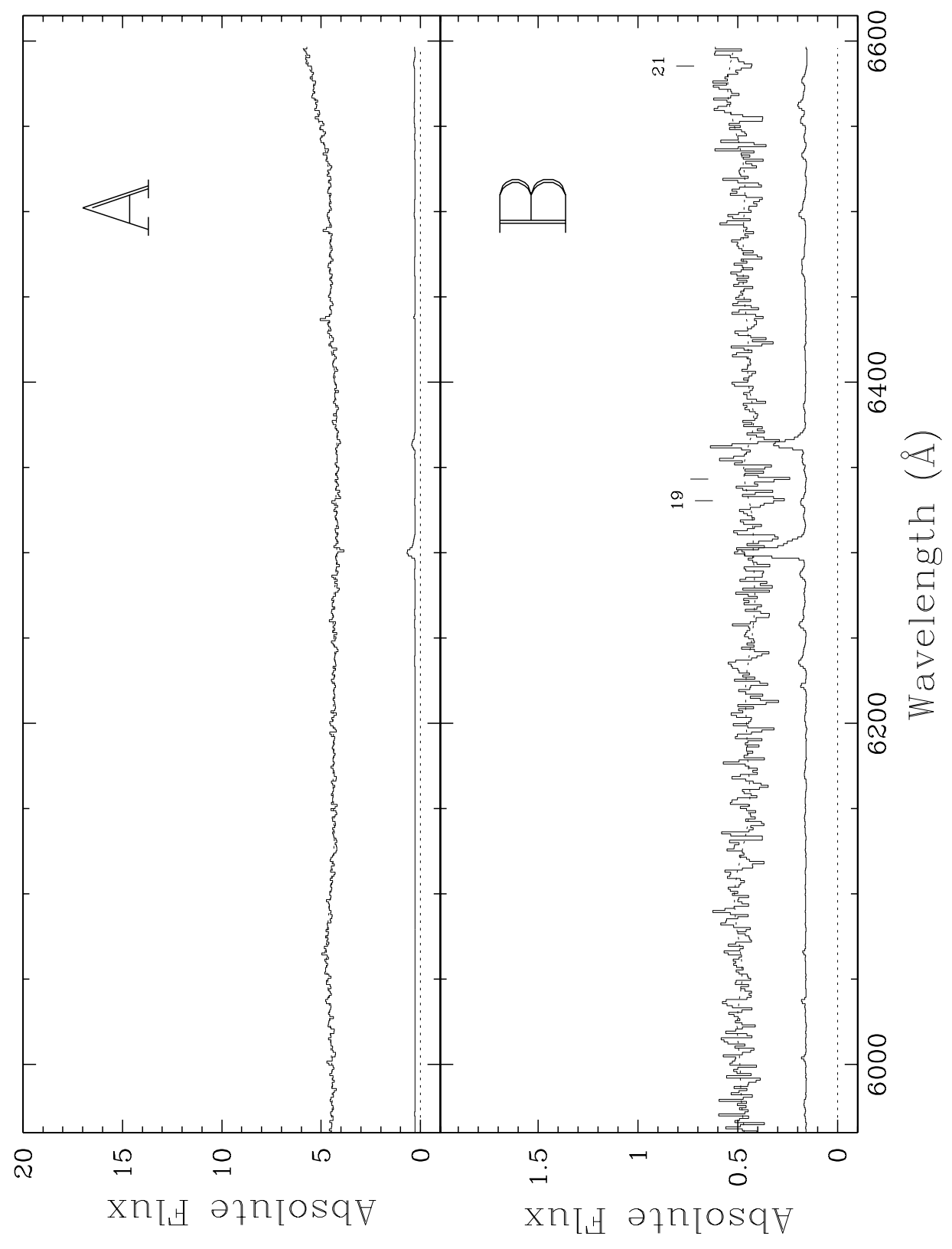




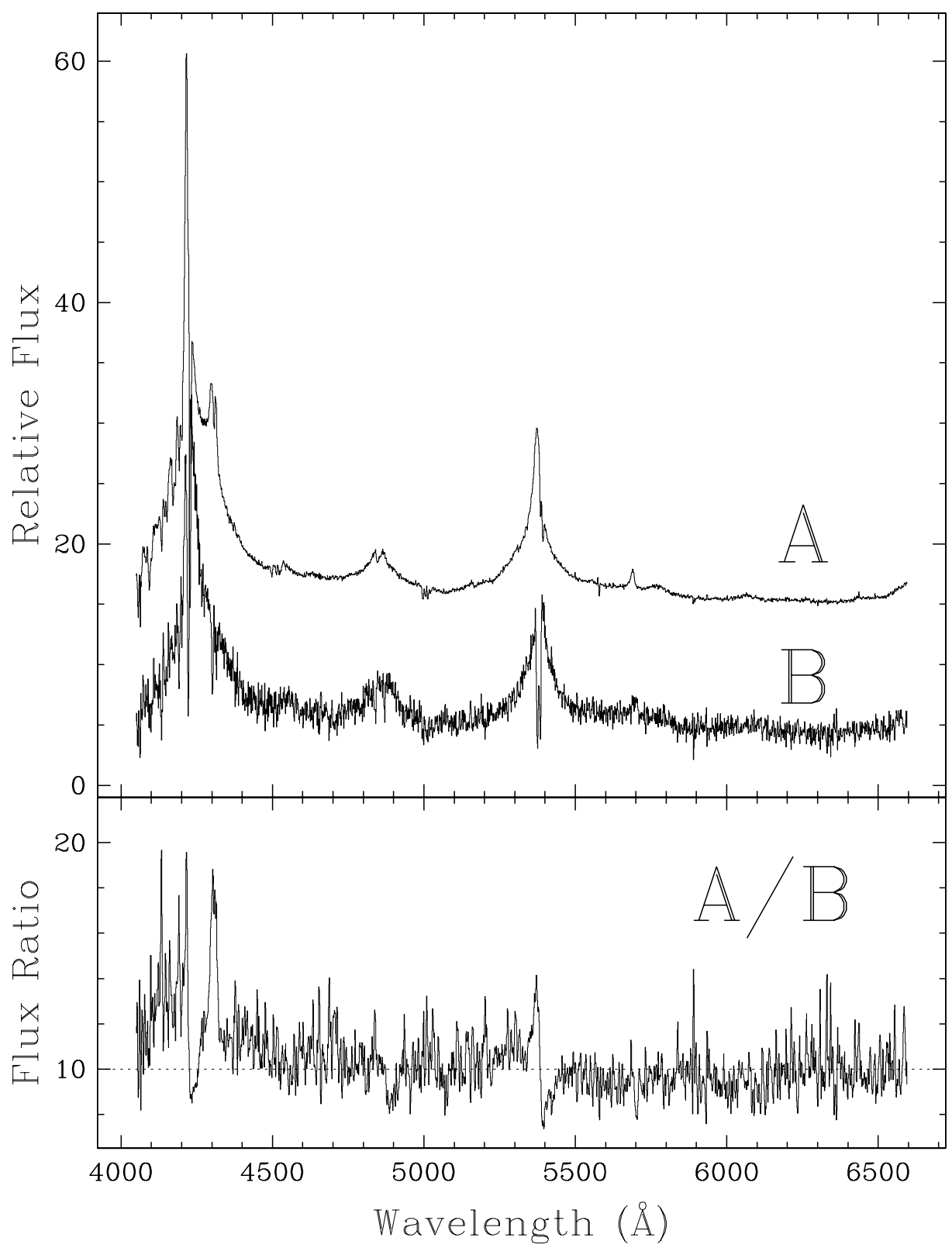

Fig. 2.- The upper panel shows the spectra of LBQS 0015+0239 components A and B scaled and offset by an arbitrary amount. The lower panel shows the ratio of component A to component $\mathrm{B}$ after interpolation over the narrow associated absorption features. The dotted line is plotted at the approximate mean flux ratio. 


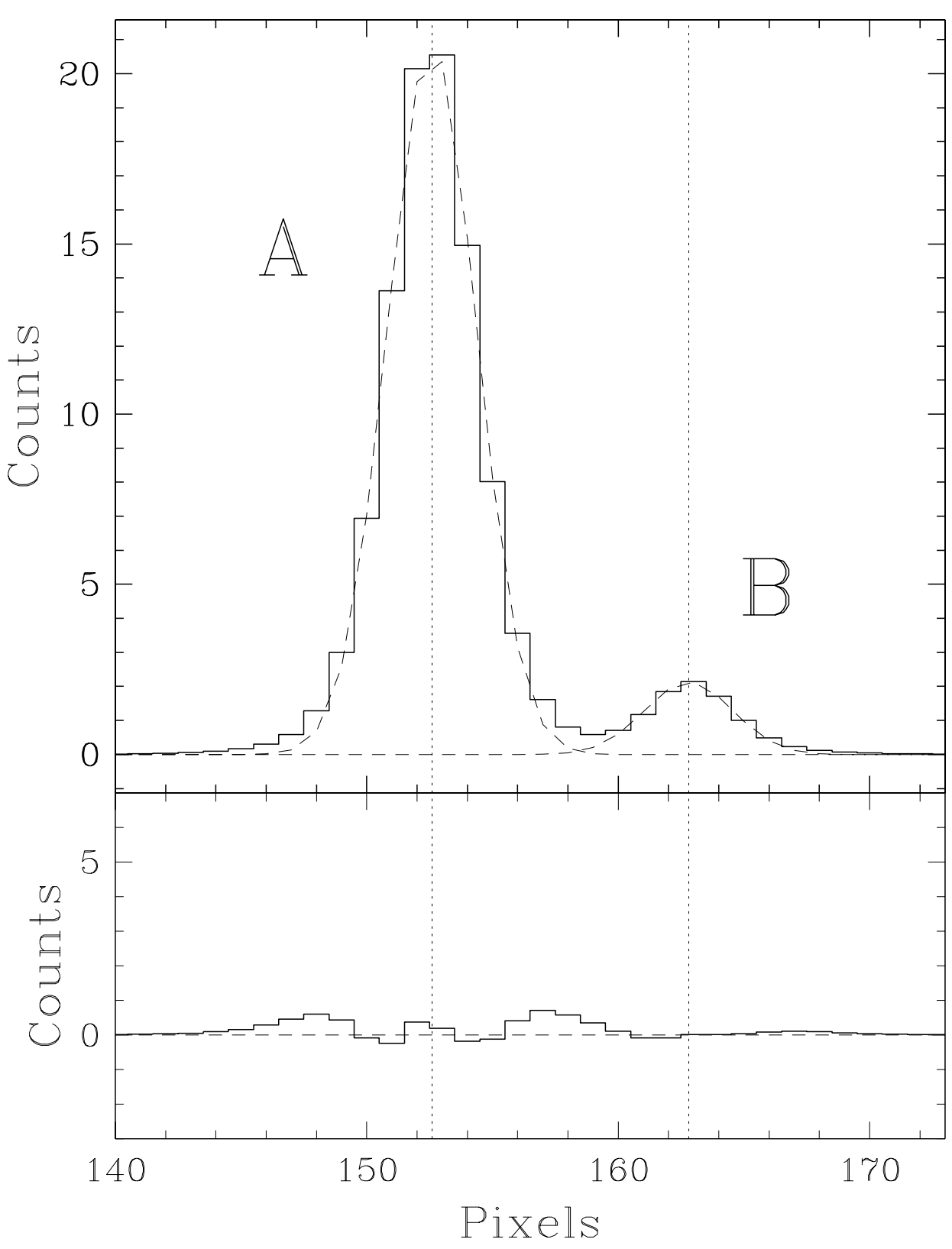

Fig. 3. - The upper panel shows the spatial cross-cut of LBQS 0015+0239 over the spec-

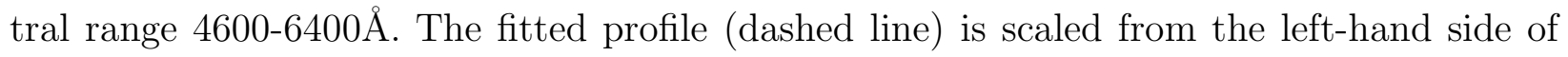
component A, where any potential light from a lensing galaxy will be negligible. The lower panel shows the residual counts after subtraction of the two fitted components. In both panels the counts are in units of $10^{4}$, and the vertical dotted lines show the centers of the two components. 
Table 1. LBQS 0015+0239 A

\begin{tabular}{|c|c|c|c|c|c|c|}
\hline Line & $\begin{array}{l}\text { Central } \lambda^{\mathrm{a}} \\
(\AA)\end{array}$ & $\begin{array}{l}\text { Equivalent Width } \\
\qquad(\AA)\end{array}$ & $\begin{array}{c}\text { FWHM }^{\mathrm{b}} \\
(\AA)\end{array}$ & $\chi_{\nu}^{2}$ & $\mathrm{SL}_{f i t}{ }^{\mathrm{c}}$ & $\mathrm{SL}_{\text {det }}{ }^{\mathrm{d}}$ \\
\hline 1 & $4056.80 \pm 0.08$ & $2.680 \pm 0.110$ & $4.37 \pm 0.21$ & 17.25 & 24.70 & 31.33 \\
\hline 2 & $4063.23 \pm 0.17$ & $2.600 \pm 0.180$ & $4.20 \pm 0.00$ & 17.25 & 14.70 & 32.92 \\
\hline 3 & $4067.52 \pm 0.68$ & $0.720 \pm 0.230$ & $4.69 \pm 1.38$ & 17.25 & 3.10 & 9.45 \\
\hline 4 & $4081.93 \pm 0.36$ & $0.570 \pm 0.090$ & $4.56 \pm 0.85$ & 2.25 & 6.20 & 7.90 \\
\hline 5 & $4093.57 \pm 0.14$ & $2.610 \pm 0.130$ & $6.12 \pm 0.37$ & 0.72 & 19.80 & 37.10 \\
\hline 6 & $4101.25 \pm 0.26$ & $1.050 \pm 0.120$ & $5.01 \pm 0.62$ & 0.72 & 8.80 & 15.97 \\
\hline 7 & $4121.04 \pm 0.71$ & $0.460 \pm 0.100$ & $6.87 \pm 1.84$ & 1.45 & 4.50 & 8.05 \\
\hline 8 & $4131.63 \pm 2.26$ & $0.640 \pm 0.590$ & $5.69 \pm 3.30$ & 1.45 & 1.10 & 11.82 \\
\hline 9 & $4134.77 \pm 0.24$ & $1.170 \pm 0.540$ & $4.20 \pm 0.00$ & 1.45 & 2.20 & 21.88 \\
\hline 10 & $4144.57 \pm 0.22$ & $0.590 \pm 0.060$ & $4.38 \pm 0.55$ & 0.35 & 9.40 & 11.52 \\
\hline 11 & $4151.71 \pm 0.13$ & $0.920 \pm 0.040$ & $4.20 \pm 0.00$ & 0.35 & 20.40 & 19.27 \\
\hline 12 & $4172.03 \pm 0.18$ & $1.950 \pm 0.120$ & $6.57 \pm 0.41$ & 1.70 & 16.70 & 48.23 \\
\hline 13 & $4179.14 \pm 0.18$ & $1.340 \pm 0.110$ & $5.32 \pm 0.38$ & 1.70 & 12.20 & 35.02 \\
\hline 14 & $4189.52 \pm 0.36$ & $0.530 \pm 0.090$ & $4.20 \pm 0.00$ & 1.55 & 6.10 & 13.12 \\
\hline 15 & $4192.63 \pm 0.19$ & $1.120 \pm 0.080$ & $4.20 \pm 0.00$ & 1.55 & 13.90 & 29.14 \\
\hline 16 & $4200.14 \pm 0.11$ & $1.920 \pm 0.060$ & $7.13 \pm 0.28$ & 1.55 & 31.20 & 55.27 \\
\hline $17^{\mathrm{e}}$ & $4228.10 \pm 0.02$ & $5.500 \pm 0.030$ & $5.76 \pm 0.04$ & 26.80 & 171.10 & 199.65 \\
\hline $18^{\mathrm{e}}$ & $4255.52 \pm 0.59$ & $0.240 \pm 0.050$ & $5.86 \pm 1.42$ & 0.72 & 4.90 & 7.05 \\
\hline 19 & $4306.59 \pm 0.12$ & $1.230 \pm 0.050$ & $6.72 \pm 0.29$ & 2.78 & 26.90 & 41.53 \\
\hline 20 & $4356.66 \pm 0.59$ & $0.200 \pm 0.050$ & $4.20 \pm 0.00$ & 1.26 & 4.30 & 4.30 \\
\hline 21 & $4382.18 \pm 0.73$ & $0.290 \pm 0.070$ & $5.98 \pm 1.77$ & 0.53 & 4.00 & 5.77 \\
\hline 22 & $4497.70 \pm 0.33$ & $0.650 \pm 0.080$ & $5.47 \pm 0.79$ & 0.77 & 8.10 & 11.13 \\
\hline 23 & $4513.08 \pm 0.34$ & $0.420 \pm 0.060$ & $4.20 \pm 0.00$ & 0.80 & 7.50 & 7.31 \\
\hline 24 & $4521.86 \pm 0.72$ & $0.360 \pm 0.130$ & $4.20 \pm 0.00$ & 0.48 & 2.70 & 6.29 \\
\hline 25 & $4526.61 \pm 1.00$ & $0.410 \pm 0.160$ & $5.38 \pm 2.17$ & 0.48 & 2.50 & 7.20 \\
\hline $26^{\mathrm{e}}$ & $4846.76 \pm 0.67$ & $0.910 \pm 0.250$ & $6.89 \pm 1.17$ & 1.06 & 3.60 & 21.07 \\
\hline 27 & $4854.93 \pm 1.54$ & $0.730 \pm 0.260$ & $9.15 \pm 2.78$ & 1.06 & 2.80 & 16.93 \\
\hline 28 & $4996.39 \pm 0.16$ & $0.960 \pm 0.070$ & $4.51 \pm 0.38$ & 1.39 & 14.00 & 17.28 \\
\hline 29 & $5004.91 \pm 0.24$ & $0.570 \pm 0.050$ & $4.20 \pm 0.00$ & 1.39 & 10.60 & 10.15 \\
\hline 30 & $5013.37 \pm 0.19$ & $0.720 \pm 0.050$ & $4.20 \pm 0.00$ & 1.07 & 13.20 & 12.76 \\
\hline
\end{tabular}


Table 1-Continued

\begin{tabular}{ccccccc}
\hline \hline Line & $\begin{array}{c}\text { Central } \lambda^{\mathrm{a}} \\
(\AA)\end{array}$ & $\begin{array}{c}\text { Equivalent Width } \\
(\AA)\end{array}$ & $\begin{array}{c}\mathrm{FWHM}^{\mathrm{b}} \\
(\AA)\end{array}$ & $\chi_{\nu}^{2}$ & $\mathrm{SL}_{f i t}{ }^{\mathrm{c}}$ & $\mathrm{SL}_{\text {det }}{ }^{\mathrm{d}}$ \\
\hline 31 & $5021.86 \pm 0.42$ & $0.380 \pm 0.070$ & $4.54 \pm 0.99$ & 1.07 & 5.30 & 6.79 \\
32 & $5166.22 \pm 0.53$ & $0.300 \pm 0.060$ & $4.20 \pm 0.00$ & 0.41 & 5.30 & 5.88 \\
33 & $5172.70 \pm 0.74$ & $0.270 \pm 0.080$ & $5.33 \pm 1.91$ & 0.41 & 3.40 & 5.27 \\
34 & $5312.80 \pm 0.53$ & $0.230 \pm 0.050$ & $4.20 \pm 0.00$ & 0.84 & 5.00 & 6.10 \\
35 & $5319.14 \pm 0.68$ & $0.260 \pm 0.070$ & $5.75 \pm 1.75$ & 0.84 & 4.00 & 7.08 \\
36 & $5332.23 \pm 0.53$ & $0.170 \pm 0.040$ & $4.20 \pm 1.27$ & 1.16 & 3.90 & 4.82 \\
37 & $5341.45 \pm 0.35$ & $0.240 \pm 0.030$ & $4.20 \pm 0.00$ & 0.36 & 7.30 & 7.12 \\
38 & $5358.85 \pm 0.77$ & $0.330 \pm 0.050$ & $9.78 \pm 1.95$ & 0.44 & 6.10 & 11.56 \\
39 & $5384.81 \pm 0.06$ & $1.280 \pm 0.040$ & $4.39 \pm 0.14$ & 2.34 & 35.10 & 50.63 \\
$40^{\mathrm{e}}$ & $5392.90 \pm 0.08$ & $1.670 \pm 0.050$ & $5.98 \pm 0.21$ & 2.34 & 35.10 & 53.67 \\
41 & $5428.49 \pm 3.05$ & $0.240 \pm 0.140$ & $11.07 \pm 7.76$ & 1.02 & 1.70 & 6.16 \\
42 & $5578.61 \pm 0.49$ & $0.510 \pm 0.120$ & $4.20 \pm 0.00$ & 2.16 & 4.20 & 5.02 \\
43 & $5889.74 \pm 0.36$ & $0.520 \pm 0.070$ & $4.20 \pm 0.00$ & 1.03 & 7.00 & 8.68 \\
44 & $5895.53 \pm 0.60$ & $0.290 \pm 0.070$ & $4.20 \pm 0.00$ & 1.03 & 4.30 & 4.85 \\
& & & & & & \\
\hline
\end{tabular}

${ }^{a}$ Wavelengths are vacuum heliocentric.

${ }^{\mathrm{b}}$ Lines with a value of $4.20 \pm 0.00$ were fit with the FWHM set to the minimum allowed value.

${ }^{\mathrm{c}}$ Significance of the line defined as $E W / \sigma_{f i t}$, where $\sigma_{f i t}$ is the error in the measured equivalent width.

${ }^{\mathrm{d}}$ Significance of the line defined as $E W / \sigma_{\text {det }}$, where $\sigma_{\text {det }}$ is the $1 \sigma$ limiting equivalent width.

${ }^{\text {e}}$ This line has been identified with an associated absorption system and is included in Table 3. 
Table 2. LBQS 0015+0239 B

\begin{tabular}{rcccccr}
\hline \hline Line & $\begin{array}{c}\text { Central } \lambda^{\mathrm{a}} \\
(\AA)\end{array}$ & $\begin{array}{c}\text { Equivalent Width } \\
(\AA)\end{array}$ & $\begin{array}{c}\mathrm{FWHM}^{\mathrm{b}} \\
(\AA)\end{array}$ & $\chi_{\nu}^{2}$ & $\mathrm{SL}_{\text {fit }}{ }^{\mathrm{c}}$ & $\mathrm{SL}_{\text {det }}{ }^{\mathrm{d}}$ \\
\hline 1 & $4055.07 \pm 0.79$ & $2.730 \pm 0.820$ & $5.56 \pm 2.02$ & 1.07 & 3.30 & 5.11 \\
2 & $4062.30 \pm 0.65$ & $2.390 \pm 0.560$ & $4.20 \pm 0.00$ & 1.07 & 4.20 & 4.42 \\
3 & $4097.22 \pm 1.21$ & $2.890 \pm 0.810$ & $8.87 \pm 2.92$ & 1.16 & 3.60 & 6.40 \\
4 & $4122.33 \pm 0.97$ & $1.590 \pm 0.530$ & $6.01 \pm 2.37$ & 0.35 & 3.00 & 4.46 \\
5 & $4133.43 \pm 0.37$ & $4.310 \pm 0.500$ & $6.68 \pm 0.94$ & 1.10 & 8.60 & 13.52 \\
6 & $4147.43 \pm 0.97$ & $2.700 \pm 0.580$ & $9.62 \pm 2.55$ & 0.92 & 4.70 & 9.13 \\
7 & $4191.85 \pm 0.32$ & $2.740 \pm 0.310$ & $5.74 \pm 0.77$ & 1.49 & 8.80 & 12.59 \\
8 & $4200.63 \pm 0.55$ & $1.170 \pm 0.210$ & $4.20 \pm 0.00$ & 1.49 & 5.50 & 6.37 \\
9 & $4204.79 \pm 0.50$ & $1.130 \pm 0.210$ & $4.20 \pm 0.00$ & 1.49 & 5.40 & 6.92 \\
$10^{\mathrm{e}}$ & $4221.22 \pm 0.58$ & $2.910 \pm 0.350$ & $4.20 \pm 0.00$ & 0.88 & 8.40 & 24.42 \\
11 & $4224.33 \pm 0.41$ & $1.930 \pm 0.440$ & $4.20 \pm 0.00$ & 0.88 & 4.40 & 16.68 \\
$12^{\mathrm{e}}$ & $4301.56 \pm 0.26$ & $2.150 \pm 0.260$ & $4.34 \pm 0.61$ & 0.64 & 8.20 & 9.91 \\
$13^{\mathrm{e}}$ & $4315.10 \pm 0.51$ & $1.180 \pm 0.300$ & $4.20 \pm 1.29$ & 1.56 & 3.90 & 4.99 \\
$14^{\mathrm{e}}$ & $4839.88 \pm 0.62$ & $1.540 \pm 0.370$ & $5.44 \pm 1.56$ & 1.21 & 4.20 & 6.03 \\
$15^{\mathrm{e}}$ & $4870.19 \pm 0.35$ & $1.560 \pm 0.210$ & $4.20 \pm 0.00$ & 0.77 & 7.40 & 7.17 \\
$16^{\mathrm{e}}$ & $5375.40 \pm 0.13$ & $6.370 \pm 0.250$ & $7.30 \pm 0.35$ & 2.42 & 25.50 & 50.60 \\
$17^{\mathrm{e}}$ & $5384.23 \pm 0.13$ & $4.080 \pm 0.220$ & $5.34 \pm 0.31$ & 2.42 & 18.60 & 33.04 \\
18 & $5712.25 \pm 1.18$ & $1.170 \pm 0.390$ & $7.29 \pm 2.83$ & 0.40 & 3.00 & 4.86 \\
19 & $6330.57 \pm 0.59$ & $1.600 \pm 0.380$ & $4.20 \pm 0.00$ & 0.44 & 4.20 & 4.55 \\
20 & $6343.12 \pm 0.48$ & $1.670 \pm 0.320$ & $4.20 \pm 0.00$ & 1.77 & 5.30 & 5.16 \\
21 & $6585.49 \pm 0.88$ & $1.120 \pm 0.380$ & $5.39 \pm 2.10$ & 0.09 & 3.00 & 4.01 \\
& & & & & & \\
\hline
\end{tabular}

${ }^{\text {a }}$ Wavelengths are vacuum heliocentric.

${ }^{b}$ Lines with a value of $4.20 \pm 0.00$ were fit with the FWHM set to the minimum allowed value.

${ }^{\mathrm{c}}$ Significance of the line defined as $E W / \sigma_{f i t}$, where $\sigma_{f i t}$ is the error in the measured equivalent width.

dSignificance of the line defined as $E W / \sigma_{\text {det }}$, where $\sigma_{\text {det }}$ is the $1 \sigma$ limiting equivalent width.

eThis line has been identified with an associated absorption system and is included in Table 3. 
Table 3. Associated Absorption

\begin{tabular}{|c|c|c|c|c|c|c|}
\hline \multirow[b]{2}{*}{ Line } & \multicolumn{2}{|c|}{ Component A } & \multicolumn{2}{|c|}{ Component B } & \multirow[b]{2}{*}{ Identification } & \multirow[b]{2}{*}{$\begin{array}{c}\Delta \mathrm{v} \\
\left(\mathrm{km} \mathrm{s}^{-1}\right)\end{array}$} \\
\hline & $\begin{array}{c}\text { Central } \lambda \\
(\AA)\end{array}$ & Redshift & $\begin{array}{c}\text { Central } \lambda \\
(\AA)\end{array}$ & Redshift & & \\
\hline 1 & $4228.10 \pm 0.02$ & 2.4780 & $4221.22 \pm 0.58$ & 2.4723 & H I 1216 & $488.2 \pm 41.2$ \\
\hline 2 & $4306.59 \pm 0.12$ & 2.4764 & $4301.56 \pm 0.26$ & 2.4723 & N V 1238 & $350.3 \pm 20.0$ \\
\hline 3 & $\ldots$ a & $\ldots$ & $4315.10 \pm 0.51$ & 2.4721 & N V 1242 & $\ldots$ \\
\hline 4 & $4846.76 \pm 0.67$ & 2.4775 & $4839.88 \pm 0.62$ & 2.4726 & Si IV 1393 & $425.9 \pm 56.5$ \\
\hline 5 & $\ldots$ b & $\ldots$ & $4870.19 \pm 0.35$ & 2.4718 & Si IV 1402 & $\ldots$ \\
\hline 6 & $5384.81 \pm 0.06$ & 2.4811 & $5375.40 \pm 0.13$ & 2.4720 & C IV 1548 & $524.3 \pm 8.0$ \\
\hline 7 & $5392.90 \pm 0.08$ & 2.4776 & $5384.23 \pm 0.13$ & 2.4720 & C IV 1550 & $482.3 \pm 8.5$ \\
\hline
\end{tabular}

${ }^{a}$ Both components of the $\mathrm{N}$ V doublet are detected in absorption in Component B. However, because $\mathrm{N} \mathrm{V}$ emission is much stronger in Component $\mathrm{A}$, ambiguities in continuum fitting have made it difficult to detect $\mathrm{N} \mathrm{V} 1242$ in absorption for this object.

b This absorption feature was detected in the spectrum of the A component, but it did not meet the significance criteria and is not included in Table 1. 